\title{
THE BRAUER GROUP OF AN AMITSUR FIELD. II CHAN-NAN CHANG
}

ABSTRACT. The structure of the Brauer group over an Amitsur field with characteristic 0 has been determined in [5]. In this paper we extend these results to the Amitsur field with characteristic $p>0$.

1. Amitsur fields. Let $K$ be a field and $K\{t\}$ be the field of all formal power series $\{t\}=\Sigma v_{v \geqslant r} a_{v} t^{\nu}, a_{v} \in K, r>-\infty$. Let $t_{1}, \cdots, t_{m}$ be a set of indeterminates over $K$. Define successively, $K_{0}=K, K_{1}=K_{0}\left\{t_{1}\right\}, \cdots, K_{m}=$ $K_{m-1}\left\{t_{m}\right\} . K_{m}$ is called an Amitsur field of $m$-indeterminates (or an Amitsur field) if $K$ is algebraically closed. Throughout this paper we assume $K_{m}^{\circ}$ is an Amitsur field with characteristic $p>0$.

The order function $o_{m}$ and the valuation ||$_{m}$ on $K_{m}$ are defined as in [5]. Similarly denote the ring of integers by $\mathcal{O}_{m}$, its unique maximal ideal by $\Re_{m}$ and the residue class field $\bar{K}_{m}=\mathcal{O}_{m} / \Re_{m} \cong K_{m-1}$.

2. The character group of $K_{m}$. Let $A$ be an abelian torsion group and let $q$ be a prime. Let $A(q)$ denote the $q$-primary component of $A$, and $A_{q}$ the subgroup of the elements in $A$ which have orders relatively prime to $q$. Let $g: A \rightarrow B$ be a homomorphism of abelian torsion groups. $g$ is said to be $q$-injective, q-surjective, $q$-isomorphism if $g$ is injective, surjective, bijective, respectively, modulo $q$-primary components. Denote a $q$-isomorphism by $\cong q$.

Let $F$ be a field. Denote the algebraic closure of $F$ by $F^{c}$ and the Galois group of $F^{c}$ over $F$ by $G(F)$. Let $L$ be an algebraic extension of $F$. We use $L_{q}$ to denote the field of all elements $x \in L$ with $[F(x): F]$ being relatively prime to $q$.

A continuous homomorphism (Krull topology) of $G(F)$ into $Q / Z$ (the rationals mod 1$)$ is called a character over $F$. We use $\chi(F)$ to denote the group of all characters over $F$. In this section we determine $\left(\chi\left(K_{m}\right)\right)_{p}$ by using induction on $m$.

Let $\hat{Z}$ be the Artin group which is the completion of $Z$ with the topolology of subgroups of finite index.

Received by the editors November 12, 1973.

AMS (MOS) subject classifications (1970). Primary 12E99, 12A60, 12 A65. 
2.1 Lemma. Let $f\{t\}=1+\Sigma_{j \geq 1} \alpha_{j} t_{m}^{j} \in K_{m-1}\left[\left[t_{m}\right]\right]$. Then $f\left\{t \in K_{m}^{n}=\right.$ $\left\{a^{n} \mid a \in K_{m}\right\}$ for every positive integer $n$ relatively prime to $p$.

Proof. By direct computation. Q.E.D.

2.2 Proposition. Let $L$ be a finite extension of $K_{m}$ with [L: $\left.K_{m}\right]$ being prime to $p$. Then there exist $T \in L$ with $T^{r}=a t_{m}$ where $a \in K_{m-1}$ and some $A$ which is algebraic over $K_{m-1}$ such that $L=K_{m}(A, T)$.

Proof. Similar to the proof of 2.2 in [5].

By induction on $m$ and 2.2, we get

2.3 Proposition. $\left(K_{m}^{c}\right)_{p}$ is generated by adjoining roots of the equations $x^{n}=t_{j}$ where $n$ is any positive integer relatively prime to $p$ and $j=1, \cdots, m$.

2.4 Theorem. $G\left(K_{m}\right) \cong_{p} \bigoplus_{j=1}^{m} \hat{Z}$.

Proof. We use $\left(K_{j}^{c}\right)_{p}$ instead of $K_{j}^{c}$ for $j=1, \cdots, m$ in the proof of 2.4 in [5]. Then the same proof works.

3. The Brauer group of the Amitsur field $K_{m}$. Denote the Brauer group of the field $L$ by $B(L)$. The following theorem of Witt [6] is well known: Let $L$ be a local field (i.e. a field which is complete in the topology induced by a discrete, nonarchimedean valuation) with residue class field $\bar{L}$. If $\bar{L}$ is perfect, then the sequence $\{1\} \rightarrow B(\bar{L}) \rightarrow B(\bar{L}) \rightarrow \chi(G(\bar{L})) \rightarrow\{1\}$ is exact and split. In case $\bar{L}$ is not perfect, the above result is no longer true. But if $L$ and $\bar{L}$ have the same characteristic, then there is a similar theorem in [9] which is stated as follow: The sequence

$$
\{1\} \rightarrow B(\bar{L}[t]) \rightarrow B(L) \rightarrow \chi(G(\bar{L})) \rightarrow\{1\}
$$

is exact and split, where $B(\bar{L}[t])$ is the Brauer group of the polynomial ring in one indeterminant $t$ over $L$. The relation between $B(\bar{L})$ and $B(\bar{L}[t])$ has been determined in $[4, \S 7]$ and may be stated as: The sequence

$$
\{1\} \rightarrow B^{\prime}(\bar{L}[t]) \rightarrow B(\bar{L}[t]) \rightarrow B(\bar{L}) \rightarrow\{1\}
$$

is exact and split, where $B^{\prime}(\bar{L}[t])$ is a subgroup of $B(\bar{L}[t])(p)$.

Hence we have $B(\bar{L}[t]) \cong_{p} B(\bar{L})$. Now we have the following proposition analogous to the theorem of Witt.

3.1 Proposition. Let $L$ be a local field with char $L=\operatorname{char} \bar{L}$ and $\bar{L}$ is not perfect. Then the sequence:

$$
\{1\} \rightarrow(B(L))_{p} \rightarrow(B(L))_{p} \rightarrow(\chi(G(\bar{L})))_{p} \rightarrow\{1\}
$$

is exact and split. 
In what follows we use induction on $m$ and 3.1 to determine the structure of $B\left(K_{m}\right)_{p}$.

3.2 Theorem. $B\left(K_{m}\right) \cong{ }_{p} \bigoplus_{i=1}^{(m-1) m / 2} Q / Z$ for all nonnegative integers $m$.

Proof. We use 3.1 instead of the theorem of Witt and $\cong_{p}$ instead of $\cong$. Then the same proof in 3.1 of [5] works.

Notations are as in $[5, \$ 4]$.

3.3 Theorem. $B\left(K_{m}\right)_{p}$ is generated by $\left\{\left(t_{i}^{r}, t_{j}\right)_{n} \mid 1 \leq i<j \leq m, 1 \leq r<n\right.$, $n$ is a positive integer which is relatively prime to $p\}$.

Proof. [5, Lemmas 4.1,4.2] are still true when $n$ is relatively prime to p. Similarly the proof of Theorem 4.3 in [5] can be carried over here.

\section{REFERENCES}

1. A. A. Albert, Structure of algebras, Amer. Math. Soc. Colloq. Publ., vol. 24, Amer. Math. Soc., Providence, R. I., 1936.

2. S. A. Amitsur, On central division algebras (to appear).

3. E. Artin, Algebraic numbers and algebraic functions, Gordon and Breach, New York, 1967. MR 38 \#5742.

4. M. Auslander and O. Goldman, The Brauer group of a commutative ring, Trans. Amer. Math. Soc. 97 (1960), 367-409. MR 22 \#12130.

5. C. N. Chang, The Brauer group of an Amitsur field, Proc. Amer. Math. Soc. 39 (1973), 493-496.

6. D. G. Northcott, An introduction to homological algebra, Cambridge Univ. Press, New York, 1960. MR 22 \#9523.

7. J.-P. Serre, Corps locaux, Actualités Sci. Indust., no. 1296, Hermann, Paris, 1962. MR $27 \# 133$.

8. E. Witt, Schiefköper über discret bewerteten Körpern, J. Reine Angew. Math. 176 (1936), 153-156.

9. S. Yuan, On the Brauer groups of local fields, Ann. of Math. (2) 82 (1965), 434-444. MR $32 \# 2449$.

DEPARTMENT OF MATHEMATICS AND STATISTICS, UNIVERSITY OF MASSACHUSETTS, AMHERST, MASSACHUSETTS 01002 